\section{SCIENCE CHINA \\ Physics, Mechanics \& Astronomy}

-News \& Views•

February 2022 Vol. 65 No. 2: 224331

https://doi.org/10.1007/s11433-021-1801-8

\title{
Ultrasound focusing with metamaterial
}

\author{
Jianchun Cheng* \\ Key Laboratory of Modern Acoustics, Ministry of Education, Institute of Acoustics, Department of Physics, Collaborative Innovation Center of \\ Advanced Microstructures, Nanjing University, Nanjing 210093, China
}

Received October 13, 2021; accepted October 21, 2021; published online December 27, 2021

Citation: J. Cheng, Ultrasound focusing with metamaterial, Sci. China-Phys. Mech. Astron. 65, 224331 (2022), https://doi.org/10.1007/s11433-021-1801-8

Ultrasonic waves, although not audible, naturally exist at high frequencies. Ultrasound can be controlled and unitized in various scenarios. Well-known examples are bats and dolphins, which produce ultrasound and detect the reflected signal for navigating and hunting. Ultrasound is also widely used in biomedical imaging and therapy as it propagates inside the human body where electromagnetic waves cannot reach without causing radiation damage. Technologies in this field have matured considerably over the last century, but the foundation of this field still primarily lies in the focusing technique. The concentration of ultrasound energy leads to high intensity in a small region, is a precondition for ultrasonic lithotripsy. Meanwhile, the compact spatial distribution also allows for imaging with a high resolution.

To avoid the complexity and large size of the equipment, metamaterial was introduced to replace the conventional active phased array [1]. By designing passive metamaterial, high-resolution acoustic focusing has been realized; however, the deep subwavelength microstructure design hinders its application in using high-frequency ultrasound in underwater detection or medical imaging [2]. Using a superoscillation technique, the frequency and spatial resolution of the ultrasonic focusing are improved, but the application is still limited due to the large side lobe [3]. In principle, highfrequency ultrasound focusing with metamaterial is challenging due to the limited space for structure design, accompanied attenuation, and mode coupling. Herein, Jiang from Fudan University and collaborators [4] demonstrate a

*Corresponding author (email: jccheng@nju.edu.cn) sparse ultrasound focusing metalens working at a frequency of up to $350 \mathrm{kHz}$ with an arbitrary focusing position and subwavelength spatial resolution. This pioneering work establishes an important milestone for using ultrasound metamaterial in medical applications.

The designed metalens consists of two types of building blocks corresponding to the ultrasound phase delays of 0 and $\pi$. More importantly, this is a sparse structure of the metalens with only one groove in each wavelength, which allows for the manipulation of high-frequency underwater sound using state-of-the-art fabrication techniques, such as the fine machining and 3D printing technologies. The extremely simple design helps discard the fine-tuning metastructure and facilitates the device fabrication process at a low cost. The theoretical design has been verified by both numerical simulation and experiments. The measured focus spot is located at the exact preset point. The focusing spatial accuracy and resolution of the metalens reach $97 \%$ and half wavelength, respectively. Meanwhile, the working frequency covers an octave bandwidth, but the radial size of the device is only 10 wavelengths. The experimental performance is close to the design limit.

The authors also explored the possibility of arbitrary focusing beyond the usual on-axis lens. To achieve this, asymmetric metasurface was designed with sparsity and preserved the original designing principle. The ultrasound can still be well focused at the designated position even if the target is off axis. This is vital because this type of metalens can be used for arbitrary position focusing. This work represents significant progress for underwater ultrasound me- 
tamaterial with a high focusing resolution, high accuracy, low cost, and large working bandwidth. Previous studies have enriched ultrasound focusing diversity by realizing the sharp autofocusing ultrasound and the ultrasound scalpel with metamaterial $[5,6]$. It will be interesting to combine these two structures for a better focusing resolution and design-related structure, such as focusing with spatial potential well and angular momentum for underwater particle control.
1 S. Zhang, L. Yin, and N. Fang, Phys. Rev. Lett. 102, 194301 (2009).

2 Y. Li, X. Jiang, R. Li, B. Liang, X. Zou, L. Yin, and J. Cheng, Phys. Rev. Appl. 2, 064002 (2014), arXiv: 1407.1138.

3 Y.-X. Shen, Y.-G. Peng, F. Cai, K. Huang, D.-G. Zhao, C.-W. Qiu, H. Zheng, and X.-F. Zhu, Nat. Commun. 10, 3411 (2019).

4 X. Jiang, J. J. He, C. X. Zhang, H. L. Zhao, W. Q. Wang, D. A. Ta, and C.-W. Qiu, Sci China-Phys. Mech. Astron. 2, 224311 (2022).

5 X. Jiang, Y. Li, D. Ta, and W. Wang, Phys. Rev. B 102, 064308 (2020).

6 J. He, X. Jiang, H. Zhao, C. Zhang, Y. Zheng, C. Liu, and D. Ta, Phys. Rev. Appl. 16, 024006 (2021). 